\title{
A Randomized Controlled Trial of High-Dose Vitamin D2 Followed by Intranasal Insulin in Alzheimer's Disease
}

\author{
Mark S. Stein ${ }^{\mathrm{a}, \mathrm{b}, *}$, Samuel C. Scherer ${ }^{\mathrm{c}, \mathrm{d}, \mathrm{e}}$, Kylie S. Ladd ${ }^{\mathrm{e}}$ and Leonard C. Harrison ${ }^{\mathrm{a}, \mathrm{b}, \mathrm{c}}$ \\ ${ }^{a}$ Royal Melbourne Hospital, Parkville, VIC, Australia \\ ${ }^{\mathrm{b}}$ Walter and Eliza Hall Institute of Medical Research, Parkville, VIC, Australia \\ ${ }^{\mathrm{c}}$ University of Melbourne, Parkville, VIC, Australia \\ ${ }^{\mathrm{d}}$ Royal Freemasons Homes of Victoria, Melbourne, VIC, Australia \\ ${ }^{\mathrm{e}}$ Cognitive, Dementia and Memory Service, Eastern Health Melbourne, Box Hill, VIC, Australia
}

\begin{abstract}
Poor vitamin D nutrition is linked with dementia, but vitamin D has not been tested in a randomized controlled trial (RCT) in Alzheimer's disease (AD). Nasal insulin acutely improves cognition and vitamin D upregulates insulin receptor expression and enhances insulin action. In an RCT we examined the effect of high-dose vitamin D followed by nasal insulin on memory and disability in mild-moderate AD. 63 community-dwelling individuals aged $>60$ were recruited; 32 with mildmoderate disease (Folstein Mini-Mental State Examination [MMSE] score 12-24) met entry criteria and were randomized. All took low-dose vitamin D (1000IU/day) throughout. After run-in ( 8 weeks), they were randomized to additional high-dose $\mathrm{D} /$ placebo for 8 weeks, followed immediately by randomization to nasal insulin (60 IU qid)/placebo for $48 \mathrm{~h}$. Primary outcome measures were Alzheimer's disease assessment scale-cognitive subscale (ADAS-cog) and Disability Assessment in Dementia (after high-dose D) and ADAS-cog and Wechsler Memory Scale-Revised Logical memory (WMS-R LM) for immediate and delayed recall (after nasal insulin). Baseline median (interquartile range, IR) age, MMSE, and ADAS-cog were 77.5 (69-80), 19.5 (17-22), and 25.5 (20-31), respectively. Median 25OHD increased from 49 to $60 \mathrm{nM}(p<0.01)$ after run-in and was 187 $\mathrm{nM}$ after high-dose vitamin $\mathrm{D}$ and $72 \mathrm{nM}$ after placebo $(p<0.001)$. Neither cognition nor disability changed significantly after high-dose D. ADAS-cog improved by a median (IR) of 9 (1-11) with nasal insulin after placebo high-dose vitamin D $(p=0.02)$, but may represent regression to the mean as WLS-R LM did not change. We conclude that high-dose vitamin D provides no benefit for cognition or disability over low-dose vitamin $\mathrm{D}$ in mild-moderate AD.
\end{abstract}

Keywords: Alzheimer's disease, nasal insulin, randomized controlled trial, vitamin D

\section{INTRODUCTION}

Dementia has been associated with poor vitamin D nutrition, and serum 25-hydroxyvitamin D (25OHD) concentration is reduced in dementia and correlates with impaired cognitive function [1-5].

\footnotetext{
Trial Registration Australian and New Zealand Clinical Trials Registry (ACTRN 12606000324516).

*Correspondence to: Mark S. Stein, The Walter \& Eliza Hall Institute of Medical Research, 1 G Royal Parade, Parkville 3052, VIC, Australia. Tel.: +61 39345 2460; Fax: +61 3 93470852; E-mail: mark.stein@mh.org.au.
}

\begin{abstract}
However, randomized controlled trials (RCTs) of vitamin $\mathrm{D}$ on cognition or memory have not been reported. Cerebrospinal fluid (CSF) insulin is reduced in moderate-severe Alzheimer's disease (AD), despite a higher concentration in plasma [6]. Intravenous insulin increases CSF insulin and acutely improves memory [7, 8] but is impractical for routine use. On the other hand, nasal insulin, which enters the CSF [9] but not the systemic circulation without absorption promoting agents [10], improves word recall and mood in healthy volunteers [11-13] and declarative memory in $\mathrm{AD}[14,15]$, but the studies of nasal insulin in AD have
\end{abstract}


not been blinded. Because vitamin D may increase insulin receptor expression and reduce insulin resistance [16-18], we hypothesized that vitamin D and nasal insulin might synergize to benefit cognition and memory in $\mathrm{AD}$. We performed an RCT to test, first, if adding high-dose vitamin D2 to ongoing low-dose vitamin D2 supplementation would improve cognition and disability in mild-moderate AD and, second, if nasal insulin immediately after high-dose vitamin D2 would further improve memory.

\section{MATERIALS AND METHODS}

\section{Feasibility pilot study}

Community dwelling participants age $\geq 60$ were conversant in English, had mild-moderate AD (Folstein Mini-Mental State Examination [MMSE] [19] score 12-24) and, if taking an anti-cholinesterase agent were on a maintenance dose $>3$ months or, having failed therapy, were off agent $>3$ months. Exclusions were coexistent cerebrovascular disease, dysphasia, abnormalities on blood screening, prior cranial surgery, epilepsy, multiple sclerosis, type 1 diabetes, renal calculi, malabsorption; poorly-controlled psychiatric disorder, alcohol intake considered excessive or any other condition that could impair compliance or cognitive assessment.

Written informed consent was obtained from participants and next of kin/carer, and the Victorian Civil and Administrative Tribunal, and the study was approved by Melbourne Health Human Research Ethics Committee.

Screening tests were performed on fasting venous blood. Plasma calcium, albumin, uric acid, and creatinine were measured on the Olympus 2700 (Olympus, Tokyo). Corrected calcium (mM) was calculated as total calcium $(\mathrm{mM})+(40$-albumin $[\mathrm{g} / \mathrm{l}]) \times 0.02$. Individuals with hypercalcemia (corrected calcium $>2.60 \mathrm{mM}$ ) or creatinine $>0.2 \mathrm{mM}$ or hyperuricemia (uric acid above the gender-matched reference range) were excluded and referred to family physicians. The serum $25 \mathrm{OHD}$ radioimmunoassay (Diasorin, Stillwater, MN) measures 25OHD2 and $25 \mathrm{OHD} 3$ [20] with coefficients of variation (CVs) at median 32, 60, and $117 \mathrm{nM}$ of 9.5, 9.5 and $9.0 \%$. PTH was measured by Immulite 2000 Intact (Siemens Los Angeles, CA) with CVs at median 3.4, 30, and $98 \mathrm{pM}$ of 8,8 and $7.5 \%$. Individuals with $25 \mathrm{OHD}>90 \mathrm{nM}$ were excluded to preserve study sensitivity [21].

For baseline assessment, a neuropsychologist recorded the ADAS-cog [22] and a geriatrician recorded the Disability Assessment in Dementia Questionnaire (DAD) based on next of kin responses [23]. Higher ADAS-cog and DAD indicate, respectively, worse cognition and less disability. There were no other assessments of subjective improvement in relatives or participants.

Treatment was open label 3000 IU vitamin D2 tablets (Cardinal Health, Braeside, Victoria) for 8 weeks, with dose adjustments to maintain 25OHD 135-160 nM.

Fasting venous blood was monitored for $25 \mathrm{OHD}$ at $1,2,3,4,6$, and 8 weeks, calcium and albumin at 4 and 8 weeks, and PTH, uric acid and creatinine at 8 weeks. After 8 weeks, ADAS-cog and DAD were repeated.

\section{Randomized controlled trial}

Inclusion and exclusion criteria were as for the pilot study, except that cerebrovascular disease was not excluded in order to broaden the clinical relevance of the RCT. Consent and ethics approval were given as for the pilot study. Blood screening was also as per the pilot study.

An occupational therapist (OT) recorded MMSE. A neuropsychologist applied ADAS-cog and Wechsler Memory Scale-Revised Logical memory (WMS-R LM) subtest for immediate and 30 minute delayed recall of two 25 information bit stories [24] and The Geriatric Depression Scale (GDS) [25]. The OT recorded, age, gender, medications, demographic, and anthropometric data, the DAD questionnaire reported by a next of kin and the Brief Pain Inventory (BPI) [26] using a verbal modification that enhances completion rate, reliability, and validity [27]. The same neuropsychologist and OT performed subsequent assessments.

Participants received one capsule daily of 1000IU vitamin D2 ('Ostelin', Boots, North Ryde, NSW) during an 8-week low-dose vitamin D run-in, following which ADAS-cog, WMS-R LM, GDS, and DAD were repeated, and venepuncture performed for the same analytes as at screening.

An off-site statistician then computer randomized to high-dose D/placebo capsules (6000 IU vitamin D2, Cardinal Health, Braeside, Victoria) blocking on AD treatment (donepezil, galantamine, none, other) (Fig. 1). Initial dosing was 2 capsules 3 times daily with food, and subsequently 0 to 2 capsules 3 times daily, reflecting empiric adjustment based on serum $25 \mathrm{OHD}$ at 2, 4, and 6 weeks, aiming to maintain 25OHD 130-175 nM. In a separate random allocation, each participant on high-dose vitamin D was paired with a 'buddy' on placebo. With every change in high- 


\section{Trial design and flow chart}
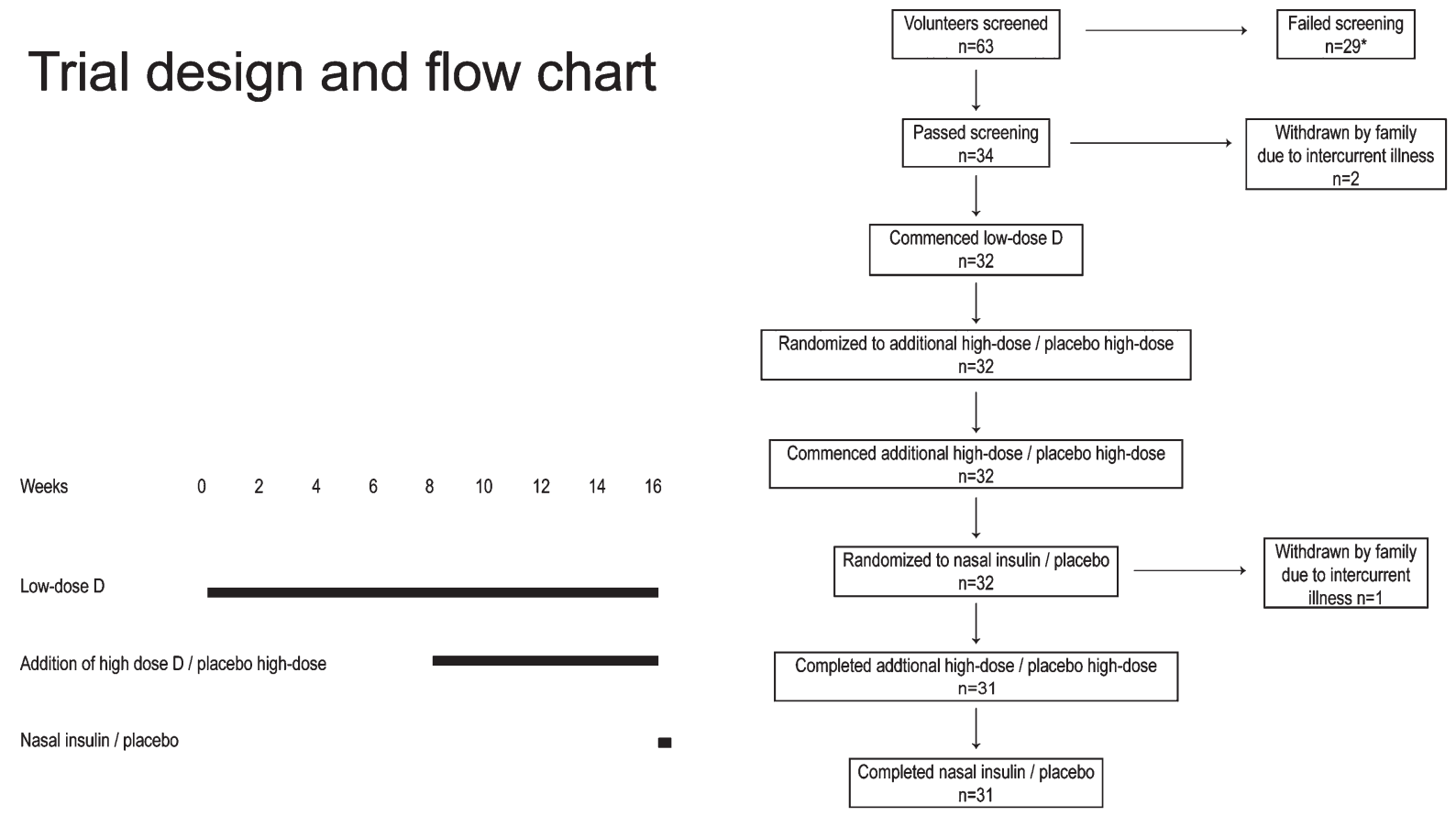

*Reasons for failure: $250 \mathrm{OHD}>90 \mathrm{nM}(\mathrm{n}=2)$, MMSE $>24(\mathrm{n}=7)$, MMSE $<12$ ( $n=5)$, vitamin $B 12$ deficiency $(n=1)$, small bowel surgery $(n=1)$, dementia not Alzheimer's disease $(n=2)$, unexpected surgery $(n=1)$ recent change in medication $(n=3)$, hyperuricaemia $(n=6)$, poor fluency in English $(n=1)$

Fig. 1. Trial design and flow chart.

dose vitamin D dose the 'buddy' was contacted to make the same capsule dose change. One investigator was unblinded to advise the pharmacist of dose adjustments. The pharmacist remained blinded and contacted participants.

All participants continued daily open-label low-dose (1000 IU) vitamin D2 as it was considered unethical for some participants to be on placebo alone given other potential benefits of $\mathrm{D}$. Thus, the randomized phase tested for benefit from additional high-dose D. D2 was used because, apart from its extra-skeletal efficacy [21], it has an additional pathway through $24 \mathrm{OHD}$ [28] and its plant origin minimizes risk of transmissible disease. Base D2, rather than the calcemic metabolite, calcitriol, permits physiologic metabolism of circulating 25OHD to a range of active metabolites [29].

Primary endpoints were ADAS-cog, WMS-R LM immediate and delayed scores, GDS, and DAD. Secondary endpoints were ADAS-cog word recognition and word recall sub-scores, and DAD sub-scores of activities of daily living.

Immediately following the randomized high-dose D/placebo phase (Fig. 1), half of each high-dose vitamin D or placebo group received nasal insulin and half nasal placebo. Randomization was stratified by MMSE $(<22$ versus $\geq 22$ ) then by drug treatment (none versus some). This allowed testing for the interaction of preceding high-dose vitamin D with nasal insulin.

Human insulin was Humulin-R (100 IU per ml, Eli Lilly, Indianapolis, IN). Placebo was Humulin-R diluent, prepared by Pharmalab (Lane Cove, NSW). Ten $\mathrm{ml}$ syringes were sterile filled with insulin or placebo by Pharmatel Fresenius Kabi (West Melbourne, Victoria). Nasal spraypump caps (code 73673; Pfeiffer, Germany) and $10 \mathrm{ml}$ brown glass bottles (code 69546; Pfeiffer, Germany) were packed by Annex (Mulgrave, Victoria), gamma irradiated (Steritech, Dandenong, Victoria), and checked for sterility by ConsulChem (Dandenong, Victoria).

Before each treatment, the syringe content was transferred into a single-use bottle and a disposable pump cap attached. The pump was primed until a uniform volume $(100 \mu \mathrm{l})$ of spray was released. Three sprays per nostril (total 60IU insulin) were administered four times daily. The same pharmacist instructed administration and supervised the first dose. Next of kin/carers were given syringes, bottles and caps, and instructions for storage and use. 
WMS-R LM story was told approximately one hour before the first nasal treatment and delayed recall recorded at approximately one hour and at one hour forty minutes after insulin. The story was repeated and delayed recall tested after a further $30 \mathrm{~min}$ and $48 \mathrm{~h}$. The story was then repeated and immediate and $30 \mathrm{~min}$ delayed recall recorded together with ADAS-cog, GDS and BPI.

Primary endpoints were ADAS-cog and WMS-R LM immediate and delayed scores. Secondary endpoints were ADAS-cog word recognition and word recall sub-scores and GDS and BPI.

\section{Statistical analysis}

Simple analyses and 95\% confidence intervals were calculated using the Minitab Release 13.1. General linear models (Minitab 13.1) for end-treatment ADAS-cog score were constructed with predictors of pre-treatment ADAS-cog score, dummy variable for randomized treatment allocation, and backward selection of clinically relevant potentially confounding variables (PTH, gender, BMI and baseline MMSE). The interaction of insulin/placebo with preceding allocation to high-dose D/placebo high-dose was tested by a general linear model. That model predicted ADAS-cog score after insulin/placebo with the following predictors: ADAS-cog score at start of insulin/placebo, dummy variables for insulin/placebo, preceding high-dose $\mathrm{D} /$ placebo, and an interaction term for the preceding high-dose vitamin D/placebo with insulin/placebo. Vitamin D and insulin analyses included all participants who completed randomized treatment. A $p$ value $<0.05$ was considered significant.

Power calculations for differences in the median change in ADAS-cog score (treatment versus placebo) were calculated with Minitab 13.1, with alpha 0.05, based on the pilot study standard deviation for change in ADAS-cog score of 3.79 ADAS-cog points. Power was $95 \%$ for a difference of 5 ADAS-cog points (highdose vitamin D versus placebo high-dose) and $80 \%$ for a difference of 4 ADAS-cog points (insulin versus placebo).

\section{RESULTS}

\section{Pilot study}

Thirteen individuals with AD, median (interquartile range [IR]) MMSE 21.5 (18.5 to 23), were studied. Median (IR) 25OHD increased from 66 (57-72) to 140 $(130-150) \mathrm{nM}(P<0.001)$ and median (IR) PTH fell from $6.3(5.6-7.0)$ to $4.4(3.5-5.6) \mathrm{pM}(P=0.012)$. No significant changes occurred in other analytes. Median (IR) baseline ADAS-cog was 25 (20-29) and median (IR) improvement in ADAS-cog score was 6.0 (4.5 to $8.5)$ points $(P<0.001)(95 \%$ confidence interval for improvement in ADAS-cog of 4.7 to 8.3 points). DAD score increased, reflecting less disability, in 11 out of $13(p<0.02)$.

\section{$R C T$}

63 community dwelling individuals with $\mathrm{AD}$ were recruited August-December 2006. 29 failed screening and two withdrew after screening (Fig. 1). 32 started treatment (Table 1): 16 took donepezil, 1 rivastigmine, 8 galantamine and 1 galantamine and memantine; 2 had type 2 diabetes treated with oral agents. All completed low-dose vitamin D run-in; 31 completed high-dose D/placebo (Fig. 1), one, on placebo, withdrawing after week 6 due to an intercurrent infection. All 31 completed insulin/placebo treatment. No other adverse events were reported.

During low dose run-in median (IR) 25OHD rose from 49 (39-67) to $60(55-70) \mathrm{nM}(P<0.01)$. Median (IR) ADAS-cog, DAD, GDS, and BPI did not change significantly between baseline and 8 weeks being, respectively, 25.5 (20-31) versus 22 (19-30), 77 (56-92) versus 74 (63-92), 2(1-5) versus $2(1-3)$, and $0(0-1)$ versus $0(0-1)$.

Groups were well matched and, with the exception of PTH (see below), did not differ significantly for any clinical or biochemical parameter in Table 2 . Nine women and 7 men were randomized to high-dose, and

Table 1

Baseline characteristics of participants

\begin{tabular}{lcc}
\hline Feature & Median & Interquartile range \\
\hline Age (years) & 77.5 & $69-80$ \\
BMI $\left(\mathrm{kg} / \mathrm{m}^{2}\right)$ & 24.5 & $22-26.5$ \\
Years of symptoms & 5 & $3-6.5$ \\
Years since diagnosis & 3 & $2-4.5$ \\
MMSE & 19.5 & $17-22$ \\
ADAS-cog & 25.5 & $20.25-30.75$ \\
WMS-RLM immediate recall & 3.5 & $2.25-6$ \\
WMS-RLM 30 min delayed & 0 & $0-0$ \\
DAD & 77 & $56-92$ \\
GDS & 2 & $1-4.75$ \\
BPI & 0 & $0-1$ \\
25OHD (nM) & 49 & $39-67$ \\
PTH (pM) & 4.3 & $3.2-5.3$ \\
Calcium (mM) & 2.48 & $2.40-2.56$ \\
Albumin (g/l) & 43.5 & $42-45$ \\
Corrected calcium (mM) & 2.40 & $2.34-2.45$ \\
Uric acid (mM) & 0.30 & $0.25-0.34$ \\
Creatinine (mM) & 0.09 & $0.08-0.10$ \\
\hline
\end{tabular}


8 women and 8 men to placebo. Median (IR) 25OHD rose from 60 (56-70) to 187 (160-240) nM on highdose vitamin D versus 64 (48-72) to 72 (63-81) nM on placebo $(p<0.001)$ (Fig. 2). The high-dose vitamin D group had a lower PTH than the placebo group (median [IR] 4.1 [3.2-5.4] versus 6.2 [4.4-7.9] pM; $p=0.046$, Kruskal Wallis). PTH fell significantly $(p=0.001)$ in both high-dose vitamin $\mathrm{D}$ and placebo groups to a median [IR] of 3.0 [2.2-3.9] and 4.9 [3.8-5.4] pM, respectively. The absolute fall in PTH was not significantly different but the proportional fall in PTH was greater in the high-dose vitamin $\mathrm{D}$ group $(P=0.025$, general linear model). Other analytes did not differ significantly within or between groups.

No significant differences in primary or secondary endpoints were detected (Fig. 3, Table 3), even after post-hoc adjustment using general linear modeling for changes in PTH, 25OHD and/or absolute levels of $25 \mathrm{OHD}$ during and/or at the end of treatment. At the start of study, 5 participants had GDS $>5$ suggesting underlying depression and, as pre-planned, were referred to primary care physicians. Post-hoc analyses excluding these participants did not affect the findings. After 8 weeks low-dose vitamin D run-in followed by 8 weeks RCT high-dose D/placebo the ADAS-cog median [IR] was 24 [19-30] for the whole study cohort. The median [IR] change in the ADAS-cog score (end RCT minus start low-dose vitamin D run-in) was 0 [ -3.0 to 4.0$]$ (95\% confidence interval for median change -2.3 to 4.0$)$.
Table 2

Characteristics of the high-dose D and placebo high-dose D cohorts at start of randomized treatment. Values are median (interquartile range)

\begin{tabular}{lcc}
\hline Feature & High-dose D & $\begin{array}{c}\text { Placebo-high } \\
\text { dose D }\end{array}$ \\
\hline Age (years) & $75(64.5-80)$ & $79(74.5-82)$ \\
BMI $\left(\mathrm{kg} / \mathrm{m}^{2}\right)$ & $24(22-26.5)$ & $25.5(22.5-28)$ \\
Years of symptoms & $4.5(3-7)$ & $5(3-7)$ \\
Years since diagnosis & $3(1.5-5)$ & $4(2-4.5)$ \\
MMSE & $19.5(17-22)$ & $19(15.5-23)$ \\
ADAS-cog & $22(19-30)$ & $22.5(18-31)$ \\
WMS-RLM immediate recall & $4(2-7)$ & $5.5(2-11)$ \\
WMS-RLM 30 min delayed & $0(0-0)$ & $0(0-2)$ \\
DAD & $71(66-88.5)$ & $76(56.5-93)$ \\
GDS & $2(1-3)$ & $2.5(1-3)$ \\
BPI & $0(0-1)$ & $0(0-1)$ \\
25OHD (nM) & $60(56-69.5)$ & $64(48-72)$ \\
PTH (pM) & $4.1(3.2-5.4)$ & $6.2(4.4-7.9)^{*}$ \\
Calcium (mM) & $2.46(2.35-2.51)$ & $2.42(2.36-2.53)$ \\
Albumin $(\mathrm{g} / \mathrm{l})$ & $41(39-43)$ & $42(40-43)$ \\
Corrected calcium (mM) & $2.40(2.32-2.49)$ & $2.39(2.36-2.43)$ \\
Uric acid (mM) & $0.30(0.27-0.35)$ & $0.29(0.25-0.34)$ \\
Creatinine (mM) & $0.09(0.08-0.09)$ & $0.09(0.08-0.10)$ \\
\hline
\end{tabular}

No significant differences in medians were detected across groups except for PTH which was different at $p=0.046$ (Kruskal Wallis test).

Eight women and 8 men were randomized to insulin, and 9 women and 7 men to placebo. Insulin and placebo groups did not significantly differ for age, BMI, number on AD pharmacotherapy, MMSE, number with MMSE $>22$ or any biochemical parameter. No signif-

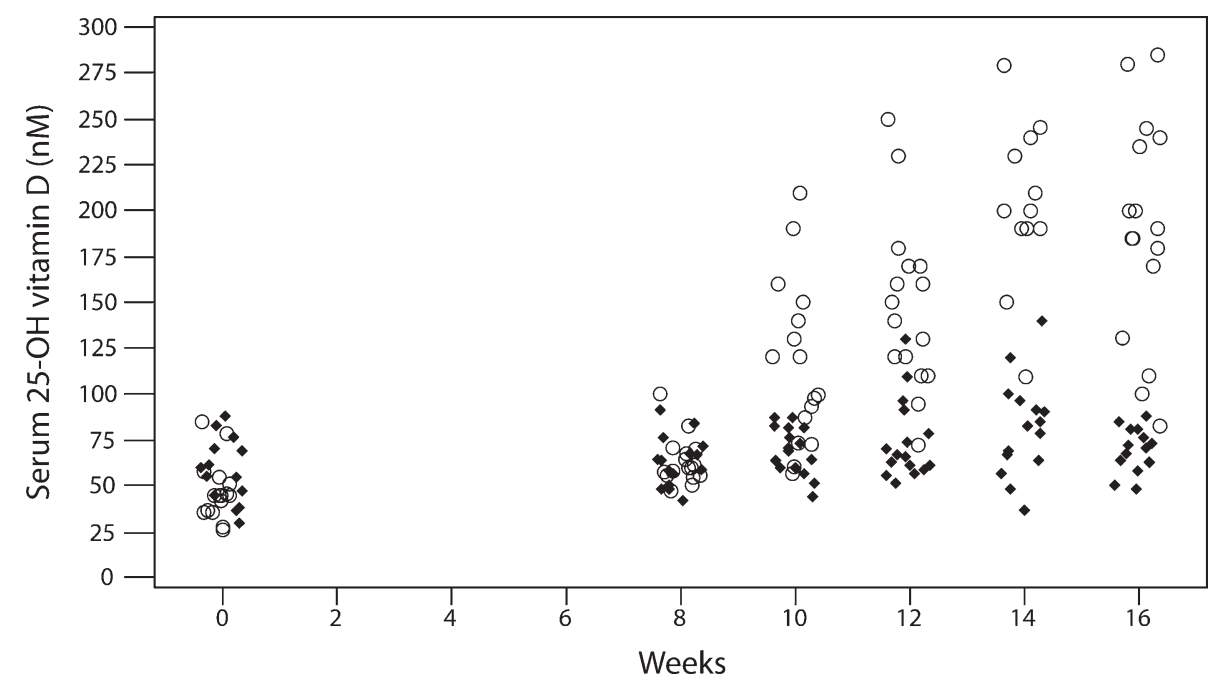

Fig. 2. Serum vitamin 25OHD during the study. All participants received low-dose vitamin D2. Circles represent those randomized to additional high-dose vitamin D2 and diamonds those randomized to placebo. Three outliers $(405,465$, and $620 \mathrm{nM}$ at week 14 falling to 240,245 , and $200 \mathrm{nM}$ end study) are not shown. 

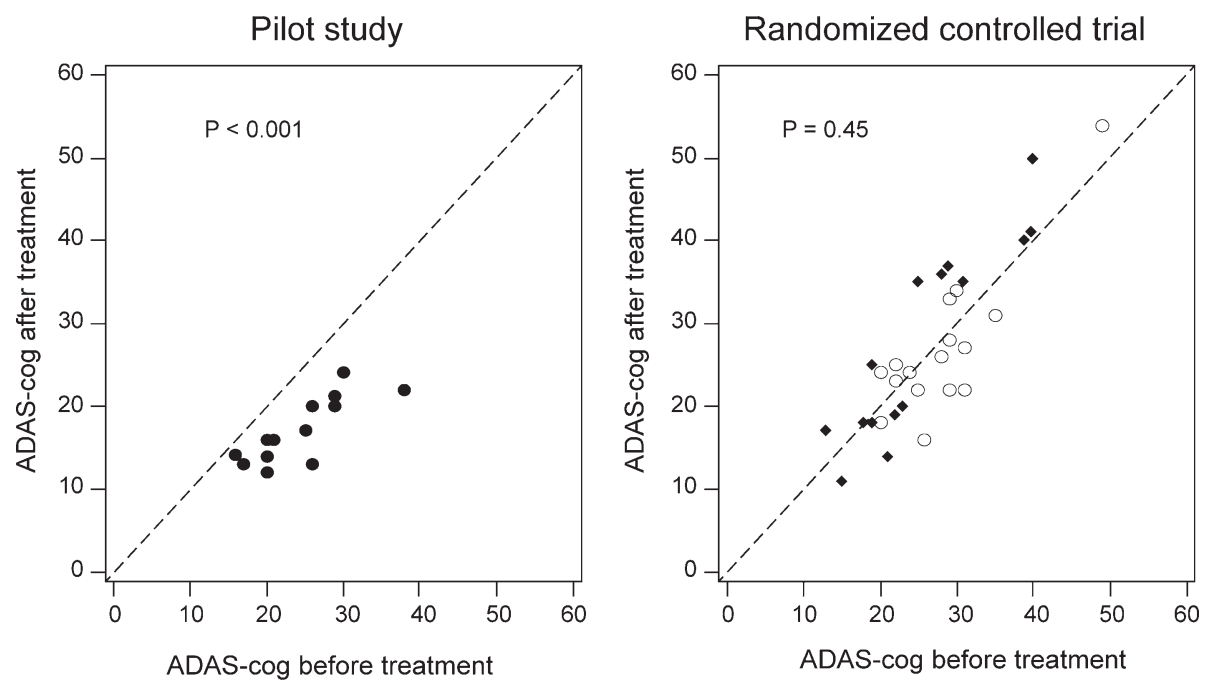

Fig. 3. Changes in ADAS-cog for the pilot study and randomized trial. Each data point represents the before (X-axis value) and after (Y-axis value) ADAS-cog scores for an individual participant. In the pilot study all points lie to right of the line of identity indicating that for every individual ADAS-cog was lower at study exit. In the randomized trial, participants who received additional high-dose vitamin D2 are plotted as circles and those who received placebo as diamonds. In the RCT, the data points are scattered around the line of identity reflecting a lack of a consistent change in ADAS-cog score.

Table 3

Changes in outcome measures with treatment

\begin{tabular}{lcc}
\hline Outcome measure & Intervention & $\begin{array}{c}\text { 95\% confidence interval } \\
\text { for the difference in median } \\
\text { changes across intervention } \\
\text { (treatment minus placebo) }\end{array}$ \\
\hline ADAS-cog & High-dose D & -5.0 to 3.0 \\
DAD & High-dose D & -7.0 to 7.0 \\
GDS & High-dose D & 0 to 2.0 \\
BPI & High-dose D & 0 to 1.0 \\
WMS-RLM immediate & High-dose D & -1 to 3.0 \\
WMS-RLM delayed & High-dose D & 0 to 1.0 \\
ADAS-cog & Nasal insulin & -6.0 to 2.0 \\
\hline
\end{tabular}

icant differences were found between the insulin and placebo groups for any endpoint.

A general linear model predicting change in ADAS$\operatorname{cog}$ score found a significant interaction between insulin/placebo with previous high-dose D/placebo $(p=0.024)$. However, this was no longer significant $(p=0.144)$ when the model was extended to take into account the ADAS-cog score at the start of insulin/placebo treatment. In a subgroup analysis, ADAS-cog changed after $48 \mathrm{~h}$ in the subgroup that received nasal insulin after placebo high-dose $\mathrm{D}$, with a median [IR] improvement of 9 [1-11] points $(p=0.02$, Mann-Whitney). However, WMS-RLM did not change significantly and statistical run-in plots suggested that this 'improvement' in ADAS-cog could have followed a chance worsening and represent regression to the mean.
Post-hoc analysis with general linear modeling restricted to those with MMSE $>22$ or according to gender or BMI revealed no benefit from nasal insulin.

\section{DISCUSSION}

This is the first double-blinded RCT to test highdose vitamin $\mathrm{D}$ and nasal insulin in AD. Despite rapid improvement in cognition and disability in an open pilot study, the RCT found no benefit from adding high-dose vitamin $\mathrm{D}$ to ongoing low-dose vitamin D supplementation. In the RCT, serum 25OHD concentrations were generally supra-physiologic and may have had no additional effect or conceivably a different effect than physiologic 25OHD concentrations in the pilot study. In addition, it is possible that differences in the rates of change of serum $25 \mathrm{OHD}$ after high-dose compared to low-dose vitamin D may have influenced the clinical outcome [30]. The RCT was powered to detect the pilot study improvement in ADAS-cog, but a small benefit cannot be excluded. By chance, PTH at the start of the RCT was lower in the high-dose vitamin D group than the placebo group. This may have made it harder to detect a benefit from high-dose vitamin D mediated through suppression of PTH. However, posthoc analyses considering changes in PTH still did not demonstrate a benefit. The fact that the entire cohort ADAS-cog score was not significantly changed after 16 
weeks of low-dose vitamin D supplementation (during 8 weeks of which half the participants were randomized to high-dose vitamin D as well) is consistent with the proposition that low-dose vitamin $\mathrm{D}$ may retard progression of $\mathrm{AD}$, which should be further tested.

Gloth and colleagues [31] reported that vitamin D improved mood and function in the elderly over one month, highlighting the potential of vitamin $\mathrm{D}$ to have rapid extra-skeletal effects. Their basal 25OHD (mean $27.5 \mathrm{nM}$ ) was low indicating many of their participants had clinical vitamin D deficiency to begin with, in contrast to our participants who started with better vitamin D nutrition. Craft et al. [8, 14] reported that intravenous and nasal insulin acutely improved memory in AD. Their participants heard two brief narratives, each containing 25 informational bits, which they were asked to recall immediately and after $10 \mathrm{~min}$ utes. We also measured recall of two 25-bit stories but found no benefit. Their definition of mild AD based on Clinical Dementia Ratings differed from ours based on MMSE. Their participants had much higher baseline recall scores than ours and probably had milder cognitive impairment, supported by the fact only 4 of 13 participants were on anti-cholinesterase therapy [14]. Thus, any benefit of nasal insulin may have been restricted to early clinical disease. The same investigators reported that memory improvement in AD was related to apolipoprotein (APO) E4 genotype and only occurred in the APOE4-negative subgroup [14, 32]. However, their sample sizes were very small for a genetic association study and they defined improvement as "percentage change from placebo". Inspection of their data (Fig. 1 in reference 14) suggests that improvement could have been due to random worsening in the placebo group. We did not stratify by ApoE as this is not routine for diagnosis or treatment and we are not aware that ApoE genotype influences responses to vitamin D.

In their nasal studies, Craft and coworkers [14, 15] used saline as placebo and mixed saline and insulin to vary the insulin dose. Double blinding would not actually be authentic without the smell of the commercial insulin diluent, and a direct chemical effect of diluent cannot be excluded. We used matched insulin diluent in a placebo-controlled RCT design and stratified insulin randomization to account for basal cognitive function. We analyzed immediate and delayed recall separately in case insulin affected these processes selectively. In addition, our design tested separate effects on recall and on the laying down of new memory. Repeat application of the same cognitive tests might have biased towards a positive finding but even with post-hoc anal- ysis that considered severity of underlying dementia, gender and BMI, we found no benefit from nasal insulin. A possible limitation is that a pharmacist supervised only the first nasal dose, subsequent doses at home being unsupervised. A nasal insulin trial of longer duration may find different outcomes.

In conclusion, despite reports that dementia is associated with poor vitamin D nutrition we found no benefit for cognition or disability from adding high-dose vitamin D to ongoing low-dose vitamin D supplementation. Furthermore, despite reports in unblinded studies of improvement in cognition with nasal insulin in $\mathrm{AD}$, we found no benefit from nasal insulin acutely or over $48 \mathrm{~h}$. We cannot exclude the possibility that an effect of high-dose vitamin D was obviated by a protective effect of low-dose D. Our findings will be important for contextualizing previous correlative studies and for meta-analyses of data from further controlled studies.

\section{ACKNOWLEDGMENTS}

Mr Eric Huggins (Chairman), Dr Peter Habersberger (Medical Director) and Trustees of The Shepherd Foundation for generous funding and enthusiastic support; Dr Mal Eutick (Pharmalab) for advice on insulin diluent; Alex Kelly (Pharmalab), David Chadwick (Pharmatel Fresenius Kabi P/L) for preparation and packaging of diluent; Richard Oppenheim, Hung Truong (Cardinal Health) for capsule manufacture; Professor Serge Gaultier, McGill University for permission to use DAD; A/Prof Ian Gordon, Statistical Consulting Centre, University of Melbourne for randomization and statistical analyses; Lynn Mather, Alzheimer's Australia Victoria, Lynne Scott and Elizabeth Fraser for recruitment; Monika Meehan for pharmacy; Vee Lyn Tan, Susan Edwards, Zoe Ellis, Caroline Francis, Jennie Barnett, Bianca Dudfield and Shinay Mackey for occupational therapy; Kate Frencham and Lucy Smith for neuropsychological assessment; Rhiannon Jones and Karen Harris for administrative assistance; Maria Bisignano, Cecilia Hsieh, Max Goodwin and Maraed Harrop for biochemical analyses. LCH is National Health and Medical Research Council Australia Senior Principal Research Fellow and is funded by a Victorian State Government Operational Infrastructure Support Grant.

Authors' disclosures available online (http://www.jalz.com/disclosures/view.php?id=860). 


\section{REFERENCES}

[1] Kipen E, Helme RD, Wark JD, Flicker L (1995) Bone density, vitamin D nutrition, and parathyroid hormone levels in women with dementia. J Am Geriatr Soc 43, 1088-1091.

[2] Martyn CN, Singh S, Wood PJ (1989) Calcium metabolism in Alzheimer's disease. A case-control study. Gerontology 35, 153-157.

[3] Sato Y, Asoh T, Oizumi K (1998) High prevalence of vitamin $\mathrm{D}$ deficiency and reduced bone mass in elderly women with Alzheimer's disease. Bone 23, 555-557.

[4] Flicker L, Mead K, MacInnis RJ, Nowson C, Scherer S, Stein MS, Thomas J, Hopper JL, Wark JD (2003) Serum vitamin $\mathrm{D}$ and falls in older women in residential care in Australia. $J$ Am Geriatr Soc 51, 1533-1538.

[5] Llewellyn DJ, Langa KM, Lang IA (2009) Serum 25hydroxyvitamin D concentration and cognitive impairment. J Geriatr Psychiatry Neurol 22, 188-195.

[6] Craft S, Peskind E, Schwartz MW, Schellenberg GD, Raskind M, Porte D, Jr. (1998) Cerebrospinal fluid and plasma insulin levels in Alzheimer's disease: relationship to severity of dementia and apolipoprotein E genotype. Neurology 50, 164168.

[7] Wallum BJ, Taborsky GJ, Jr., Porte D, Jr., Figlewicz DP, Jacobson L, Beard JC, Ward WK, Dorsa D (1987) Cerebrospinal fluid insulin levels increase during intravenous insulin infusions in man. J Clin Endocrinol Metab 64, 190194.

[8] Craft S, Newcomer J, Kanne S, Dagogo-Jack S, Cryer P, Sheline Y, Luby J, Dagogo-Jack A, Alderson A (1996) Memory improvement following induced hyperinsulinemia in Alzheimer's disease. Neurobiol Aging 17, 123-130.

[9] Born J, Lange T, Kern W, McGregor GP, Bickel U, Fehm HL (2002) Sniffing neuropeptides: a transnasal approach to the human brain. Nat Neurosci 5, 514-516.

[10] Moses AC, Gordon GS, Carey MC, Flier JS (1983) Insulin administered intranasally as an insulin-bile salt aerosol. Effectiveness and reproducibility in normal and diabetic subjects. Diabetes 32, 1040-1047.

[11] Kern W, Born J, Schreiber H, Fehm HL (1999) Central nervous system effects of intranasally administered insulin during euglycemia in men. Diabetes 48, 557-563.

[12] Benedict C, Hallschmid M, Hatke A, Schultes B, Fehm HL, Born J, Kern W (2004) Intranasal insulin improves memory in humans. Psychoneuroendocrinology 29, 1326-1334.

[13] Benedict C, Kern W, Schultes B, Born J, Hallschmid M (2008) Differential sensitivity of men and women to anorexigenic and memory-improving effects of intranasal insulin. J Clin Endocrinol Metab 93, 1339-1344.

[14] Reger MA, Watson GS, Frey WH, 2nd, Baker LD, Cholerton B, Keeling ML, Belongia DA, Fishel MA, Plymate SR, Schellenberg GD, Cherrier MM, Craft S (2006) Effects of intranasal insulin on cognition in memory-impaired older adults: modulation by APOE genotype. Neurobiol Aging 27, 451-458.

[15] Reger MA, Watson GS, Green PS, Wilkinson CW, Baker LD, Cholerton B, Fishel MA, Plymate SR, Breitner JC, DeGroodt W, Mehta P, Craft S (2008) Intranasal insulin improves cognition and modulates beta-amyloid in early AD. Neurology 70, 440-448.

[16] Chaplinski TJ, Bennett TE (1987) Induction of insulin receptor expression of human leukemic cells by 1 alpha, 25 dihydroxyvitamin D3. Leuk Res 11, 37-41.
[17] Maestro B, Davila N, Carranza MC, Calle C (2003) Identification of a Vitamin D response element in the human insulin receptor gene promoter. J Steroid Biochem Mol Biol 84, 223230.

[18] von Hurst PR, Stonehouse W, Coad J (2010) Vitamin D supplementation reduces insulin resistance in South Asian women living in New Zealand who are insulin resistant and vitamin D deficient - a randomised, placebo-controlled trial. Br J Nutr 103, 549-555.

[19] Folstein MF, Folstein SE, McHugh PR (1975) "Mini-mental state". A practical method for grading the cognitive state of patients for the clinician. J Psychiatr Res 12, 189-198.

[20] Hollis BW (2000) Comparison of commercially available (125) I-based RIA methods for the determination of circulating 25-hydroxyvitamin D. Clin Chem 46, 1657-1661.

[21] Flicker L, MacInnis RJ, Stein MS, Scherer SC, Mead KE, Nowson CA, Thomas J, Lowndes C, Hopper JL, Wark JD (2005) Should older people in residential care receive vitamin D to prevent falls? Results of a randomized trial. J Am Geriatr Soc 53, 1881-1888.

[22] Rosen WG, Mohs RC, Davis KL (1984) A new rating scale for Alzheimer's disease. Am J Psychiatry 141, 1356-1364.

[23] Gelinas I, Gauthier L, McIntyre M, Gauthier S (1999) Development of a functional measure for persons with Alzheimer's disease: the disability assessment for dementia. Am J Occup Ther 53, 471-481.

[24] Wechsler. (1987) Wechsler Memory Scale-Revised Manual. The Psychological Corporation, New York.

[25] Yesavage JA (1988) Geriatric Depression Scale. Psychopharmacol Bull 24, 709-711.

[26] Cleeland (1989) Measurement of pain by subjective report. In Issues in pain measurement, Chapman CR, Loeser JD, eds, Raven Press, New York.

[27] The Australian Pain Society (2005) Pain in Residential Care Facilities: Management Strategies, http://www.apsoc.org.au/ owner/files/9e2c2n.pdf, Accessed Jan 13.

[28] Mawer EB, Jones G, Davies M, Still PE, Byford V, Schroeder NJ, Makin HL, Bishop CW, Knutson JC (1998) Unique 24-hydroxylated metabolites represent a significant pathway of metabolism of vitamin D2 in humans: 24-hydroxyvitamin D2 and 1,24-dihydroxyvitamin D2 detectable in human serum. J Clin Endocrinol Metab 83, 2156-2166.

[29] Garcion E, Wion-Barbot N, Montero-Menei CN, Berger F, Wion D (2002) New clues about vitamin D functions in the nervous system. Trends Endocrinol Metab 13, 100-105.

[30] Vieth R (2004) Enzyme kinetics hypothesis to explain the Ushaped risk curve for prostate cancer vs. 25-hydroxyvitamin $\mathrm{D}$ in nordic countries. Int $J$ Cancer 111, 468.

[31] Gloth FM, 3rd, Smith CE, Hollis BW, Tobin JD (1995) Functional improvement with vitamin D replenishment in a cohort of frail, vitamin D-deficient older people. J Am Geriatr Soc 43, 1269-1271.

[32] Craft S, Asthana S, Cook DG, Baker LD, Cherrier M, Purganan K, Wait C, Petrova A, Latendresse S, Watson GS, Newcomer JW, Schellenberg GD, Krohn AJ (2003) Insulin dose-response effects on memory and plasma amyloid precursor protein in Alzheimer's disease: interactions with apolipoprotein E genotype. Psychoneuroendocrinology 28, 809-822. 